\title{
Evaluation des Hauptlesesaals
}

\section{Vorbereitungen für die WLB der Zukunft}

Nach vielen Jahren der beengten Raumsituation mit geringem Veränderungspotential in der Bibliothek lassen nun der Erweiterungsbau und die Sanierung des Bestandsgebäudes zukunftsweisende Überlegungen und konkrete Planungen für eine Neugestaltung der Benutzungsbereiche zu. Mit der räumlichen Erweiterung der Bibliothek können in Zukunft zusätzliche Flächen für Arbeitsplätze in unterschiedlicher Ausgestaltung, aber auch weitere Freihand- und Präsenzbestände sowie eine verbesserte technische Infrastruktur angeboten werden. Grundlegende Frage für alle anstehenden Planungen ist hierbei, wie die Aufteilung zwischen Präsenzbeständen und Benutzerarbeitsplätzen optimal gestaltet werden soll. Ist ein gut ausgebauter Präsenzbestand im Zeitalter des Internets und der elektronischen Medien für die Zukunft noch im Interesse der Benutzerinnen und Benutzer?

Um diesen Entscheidungsfindungsprozess für eine bedarfsorientierte Aufteilung und optimale räumliche Strukturierung der Flächen nach Zonen für konzentriertes bzw. kommunikatives Lernen sowie Pausenbereiche anzustoßen, wurden für den Hauptlesesaal die Medienbestände, technische Ausstattung sowie der Bedarf für die Zukunft in verschiedenen Prozessen evaluiert und in differenzierter Sichtweise analysiert. Hierfür sind im Jahr 2017 die Fachreferenten, die Benutzer der WLB und zusätzlich die Benutzungsleiter der großen wissenschaftlichen Bibliotheken des Landes in unterschiedlicher Weise befragt worden, um einen Gesamteindruck zu gewinnen. Die aus allen Befragungen gewonnenen Daten und Kommentare sind Auftakt für weitere interne Diskussionen und Planungen, die bereits für die konkrete Ausgestaltung der neuen und der sanierten Räumlichkeiten begonnen haben.

Damit alle Planungen sich auf eine solide Basis stützen können, ist dieser Entscheidungsfindungsprozess in verschiedenen Phasen und auch aus unterschiedlicher Sichtweise angestoßen worden. Zunächst war eine wesentliche Voraussetzung, den Ist-Zustand in Abstimmung mit den Fachreferenten zu erfassen und daraus entsprechenden Diskussionsbedarf abzuleiten.

Im Dezember 2017 folgte im Rahmen einer Projektarbeit einer Studentin der HdM Stuttgart eine Benutzerbefragung in der WLB. Diese Befragung ist als wichtiger Bestandteil zu sehen, um die derzeitige Situation mit den Interessen und Anliegen der Benutzer abzugleichen und somit für die Zukunft eine breite, fundierte Planungsgrundlage zu gewinnen.

Ziel aller Analysen ist es, den Service für die Benutzer zu verbessern und in den entsprechenden Bau- und Sanierungsphasen das Profil und Angebot des Hauptlesesaals bedarfsgerecht für die Zukunft zu gestalten. Dabei ist zu berücksichtigen, dass in der WLB keine homogene Benutzergruppe vertreten ist, sondern vielschichtige wissenschaftliche Interessen, berufliche Erfordernisse und auch private Forschungsschwerpunkte Berücksichtigung finden. Diese Breite und Vielfalt spiegelt sich auch im Bestand und in den Angeboten des Hauptlesesaals wider, der in einer Universalbibliothek (weitgehend) alle Fächer - wenn auch mit unterschiedlichen Schwerpunkten und Bestandsgrößen - abdeckt. ${ }^{1}$

Durch den Prozess der Analysen der Angebote des Hauptlesesaals wurden wertvolle und richtungsweisende Aussagen gewonnen, die für die weiteren Zukunftsplanungen und Aufgaben der Bibliothek zielführend sind. Die praktische Umsetzung wird fortgeführt, soweit es die konkrete Ausgestaltung für den Service im Erweiterungsbau und die Planungen für das sanierte Bestandsgebäude in den zeitlich entsprechenden Bauabschnitten zulassen. Hier können nur die wesentlichen Aspekte und Ergebnisse der Befragungen beschrieben werden. 


\section{Ist-Analyse des Hauptlesesaals}

Auftakt für alle weiteren Überlegungen war die Befragung der Fachreferenten im Frühjahr 2017, um die derzeitige Situation zu erfassen.

\subsection{Profil des Präsenzbestands}

Wie in allen großen wissenschaftlichen Allgemeinbibliotheken des Typs Staats- und Landesbibliotheken hat auch die WLB einen, Allgemeinen Lesesaal'. ${ }^{2}$ Dieser zeichnet sich dadurch aus, dass hier Literatur zu (nahezu) allen Fachgebieten zu finden ist. ${ }^{3}$ Mit 53.102 Bänden ${ }^{4}$ entspricht diese Größenordnung dem Bestand, der auch in anderen großen Bibliotheken des gleichen Typs aufgestellt ist. Der Präsenzbestand zu Baden-Württemberg mit 7.449 Bänden kann als eine Art Fachabteilung im Hauptlesesaal gesehen werden. Die Kunst mit ca. 10.000 Bänden befindet sich derzeit aus Platzgründen noch im Sonderlesesaal, soll aber mit der räumlichen Vergrößerung des Hauptlesesaals in den Bestand integriert werden. Dies ist auch für den kleinen Restbestand von 1.470 Bänden an Bibliografien und Adressbüchern der HB Information geplant.

Der Präsenzbestand ist nur beschränkt ausleihbar, d.h. zu den Schließzeiten der Bibliothek über Nacht sowie an Wochenenden bzw. Feiertagen. Die systematische Aufstellung ermöglicht einen geordneten, strukturierten Zugang zu den Fächern. Derzeit werden die Präsenzbestände nach der Regensburger Verbundklassifikation (RVK) umgearbeitet, da absehbar ist, dass die alte Aufstellungssystematik bei einer Vergrößerung des Bestandes nicht ausreichen wird.

In einem wissenschaftlichen Lesesaal finden sich im Präsenzbestand typischerweise Nachschlagewerke aller Art wie Enzyklopädien, Lexika, ein- und mehrsprachige Wörterbücher, Sachwörterbücher, Verzeichnisse aller Art für Daten und Fakten wie Statistiken, biografische Nachschlagewerke und Register. Weitere Werke sind je nach Fachgebiet Einführungen, Lehrbücher, Handbücher, Quellentexte, Textausgaben, Kommentare und Zeitschrif-

2) Vgl. dazu: Hilpert, Wilhelm u.a.: Benutzungsdienste in Bibliotheken: Bestands- und Informationsvermittlung, Berlin 2014, S. 139-141.

3) Der Sonderlesesaal ergänzt mit seinen Nachschlagewerken die Präsenzbestände zu den Handschriften, Alten u. Wertvollen Drucken, der Kunst sowie den Karten und Grafiken. Dieser Bestand umfasst ca. 32.500 Bände.

4) Stand Ende 2017.

5) Vgl. dazu: Ganter, Klaus: Bibliothekarisches Grundwissen, 9. Auflage, Berlin 2016, S. 260 ten. Eine Zeitungsauslage und Semesterapparate für örtliche Hochschulen sind integriert.

Diese Auswahl entspricht den Kriterien nach Inhalt und Nutzung der Medien. Zu einem gut sortierten Präsenzbestand gehören typischerweise: ${ }^{5}$

- Nachschlagewerke mit kurzen übersichtlichen Texten zur sachlichen Erstinformation als Einstieg in die wissenschaftlichen Fachgebiete sowie

- Werke, die von vielen Benutzern kurzfristig bzw. häufig benötigt werden und daher vor Ort vorhanden sein sollten.

Bei stark nachgefragten Werken wie Einführungen und Lehrbüchern wird in Auswahl ein Mehrfachexemplar für die Magazinausleihe beschafft, soweit die jeweiligen Etats der Fächer ein Zweitexemplar zulassen.

\subsection{Bestandsgröße der Fächer}

Die Analyse nach der Bestandsgröße der Fachgebiete ergibt vier Bestandsgruppen mit folgender Verteilung:

- 6 „kleinste" Fächer mit bis zu 150 Bänden (Umwelt, Bibliothekswesen, Sonstige Sprachen, Astronomie, Sport, Buch- und Verlagswesen),

- 10 "kleine" Fächer mit 151 bis 300 Bänden (Informatik, Physik, Massenmedien, Psychologie, Mathematik, Politik, Erziehungswissenschaften, Slawistik, Technik, Landwirtschaft/Ernährungswissenschaft),

- 16 „mittlere" Fächer mit 301 bis 2.000 Bänden (Soziologie/Statistik, Tanz/Film/Theater, Ethnologie, Allgemeine Naturwissenschaften, Geowissenschaften, Wissenschaft/Hochschulwesen, Klassische Philologie/Latein, Allgemeine Sprachwissenschaft, Romanistik, Chemie, Anglistik/ Germanische Sprachen, Biologie, Archäologie/ Alte Geschichte, Philosophie, Geographie, Germanistik) sowie

- 9 "große" Fächer mit mehr als 2.000 Bänden (Sprachwörterbücher, Medizin, Allgemeine Nachschlagewerke, Wirtschaftswissenschaften, Geschichte/Zeitgeschichte, Theologie, Jura, Musik, Baden-Württemberg). 
Die graphische Darstellung (Abb. 1) macht u.a. deutlich, dass die neun „großen“ Fächer knapp drei Viertel des Platzangebots im Hauptlesesaal belegen. Sie gehören im Profil der Bibliothek zu den Sammelschwerpunkten aufgrund der Ausrichtung der Bibliothek nach ihren besonderen Altbeständen, ihrer Benutzerstruktur und der großen Nachfrage in den „studentenstarken" Fächern.

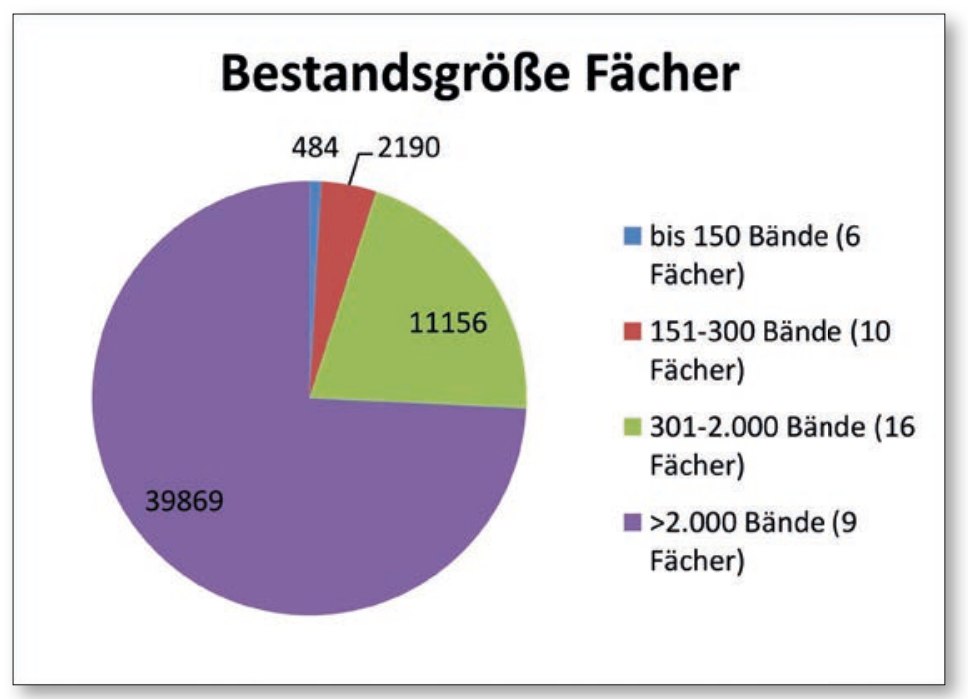

Abb. 1: Bestandsgröße der Fächer im Hauptlesesaal

\subsection{Befragung der Fachreferenten}

Anfang 2017 wurden die Fachreferenten anhand eines Fragebogens nach ihren Bestandsprofilen und Auswahlkriterien der Präsenzbestände für ihre Fachgebiete befragt.

\subsubsection{Auswahl der Präsenzbestände}

Die Auswertung nach der Auswahl der Titel für den Präsenzbestand ergab folgende Aussagen:

- Die inhaltliche Aktualität (neue Themen) des Fachgebiets ist für alle Fächer wichtig.

- Nur ein Teil der Titel, die Benutzer stark nachfragen, findet sich auch im Hauptlesesaal. Eine hohe Nachfrage sollte zur Anschaffung von Mehrfachexemplaren führen.

- Die Auswahl ist weitgehend nicht auf besondere Zielgruppen ausgerichtet, sondern berücksichtigt Benutzer mit unterschiedlichem Interesse.

- Die Fächer werden mit Literatur in der Breite der Fachdisziplinen aufgebaut. Nur bei den größeren Fächern mit mehr Bestand können zusätzlich Schwerpunkte gesetzt werden.
Für alle Aussagen zeigen sich Einschränkungen vor allem auch dadurch, dass aus Platzgründen nur eine Auswahl der relevanten Titel im Hauptlesesaal aufgestellt werden kann. Bei manchen Fächern ist bereits jetzt zu wenig Platz vorhanden und eine Erweiterung dringend geboten. Insbesondere die historisch ausgerichteten Fächer müssen darauf achten, dass ältere Quellen vorhanden sind, aber auch neue aktuelle Titel hinzukommen. In den meisten Fächern haben sich neue Forschungsschwerpunkte gebildet, die zusätzlichen Stellplatz benötigen. Für acht Fächer ergab die Rückmeldung der Fachreferenten, dass der Platz bereits jetzt nicht ausreicht. Zu diesen Fächern gehören die Massenmedien, Germanistik, Philosophie, Allgemeine Nachschlagewerke, Wirtschaftswissenschaften, Theologie, Jura und Baden-Württemberg. Allein für die Kunst und Musik wurde eine Reduzierung der Präsenzbestände genannt. ${ }^{6}$ Der Bestand der Musik wurde bereits deutlich reduziert. Die Kunstbände können erst in den Hauptlesesaal integriert werden, wenn zusätzlicher Platz vorhanden sein wird.

Die räumliche Situation des Hauptlesesaals ist derzeit so beengt, dass keine zusätzlichen Flächen für eine Erweiterung der Präsenzbestände vorhanden sind, da Benutzerarbeitsplätze sowie Scanner- und Reproduktionsplätze aufgrund der großen Nutzung nicht reduziert werden können. Dies bedeutet, dass die Kapazitäten in allen Bereichen bereits voll ausgeschöpft und Veränderungen nicht ohne weiteren Platzbedarf möglich sind.

\subsubsection{Entwicklung der Präsenzbestände}

Die Pflege der Präsenzbestände nach den Anforderungen und der Weiterentwicklung der jeweiligen Fachgebiete gehört zu den wichtigen Aufgaben der Fachreferenten. Die Auswertung der Fragebögen ergab folgende Aussagen:

- In allen Fächern werden ältere Auflagen durch Neuerscheinungen ersetzt. Der Lesesaalbestand bildet somit den aktuellen Stand in den Disziplinen ab.

- „Klassiker des Fachs“ (d.h. ältere Titel) sind überwiegend nur für die geistes- und sozialwissenschaftlichen Fächer relevant.

6) Für beide Fächer war mit dem Bezug des Bestandsgebäudes ein eigener Fachlesesaal eingerichtet worden. Diese Lesesäle mussten aus räumlichen und personellen Gründen jedoch aufgelöst werden, so dass die Bestände in den Hauptlesesaal (Musik) bzw. den Sonderlesesaal (Kunst) integriert wurden. Mit dieser Integration wurde eine Reduzierung des Bestands der ursprünglichen Fachlesesäle notwendig. 
- Diskussionsbedarf besteht bei der Frage, ob bei Nachschlagewerken elektronische Ausgaben den Vorzug erhalten sollten. Aus Platzgründen werden die gedruckten Ausgaben häufig ins Magazin umgestellt.

\subsection{Best practice in anderen Bibliotheken}

Um das Meinungsbild für die internen Diskussionen zu erweitern, wurden über die E-Mail-Gruppe $\operatorname{der} A G$ Benutzung auch die Benutzungsleiter der anderen großen wissenschaftlichen Bibliotheken im Land befragt. Bei den Universitätsbibliotheken zeigt sich bereits ein deutlicher Unterschied dadurch, dass diese über eine ausgebaute Lehrbuchsammlung mit Mehrfachexemplaren verfügen. Ein Exemplar des Lehrbuchs befindet sich dabei auch im Präsenzbestand. Die Fächer mit der größten Nachfrage sind meist Jura, Wirtschaftswissenschaften und Medizin.

Wie in der WLB werden Nachschlagewerke zunehmend in elektronischer Form angeschafft. Überwiegend bleibt bei Parallelausgaben das gedruckte Werk im Präsenzbestand. Ausnahmen bestehen, wenn die Exemplare nur in elektronischer Form erworben werden, damit aber auch in den Räumen der Bibliothek verfügbar sind. Da auch die Versorgung der nicht-universitären Benutzer je nach Lage und Größe der Bibliothek eine bedeutende Rolle spielt, ist darauf zu achten, dass durch E-Ressourcen keine Benutzer in der Zugriffsberechtigung ausgeschlossen werden.

Es stellt sich generell die Frage, wie begrenzte Räumlichkeiten optimal eingerichtet werden. Neben den Präsenzbeständen besteht ein großer Bedarf an Arbeitsplätzen und Lernangeboten.

\section{Benutzerbefragung zum Hauptlesesaal}

Nachdem die letzte Benutzerbefragung zum Hauptlesesaal bereits 10 Jahre zurückliegt, sollten wiederum neue Aussagen aus Sicht der Benutzer gewonnen werden, um aktuelle Daten für weitere Planungen zu gewinnen. Diese Projektarbeit wurde von Frau Claudia Exner (Hochschule der Medien Stuttgart) im Rahmen ihres Praxissemesters 2017/18 an der WLB übernommen.
Die Umsetzung der Befragung erfolgte vom 6. bis zum 19. Dezember 2017. Zuvor fand ab dem 23. November 2017 eine intensive Werbephase über die Website (Startseite, Aktuelles, Hauptlesesaal), Facebook und einen Hinweis im OnlineKatalog statt. In den Räumen der Bibliothek wurde an den beiden Hauseingängen und am Eingang zum Hauptlesesaal über Plakate (mit Link und QR-Code) sowie über Infostele und Flyer entsprechend geworben.

\subsection{Fragebogen}

Für die Fachreferenten ist es nicht einfach, genaue Angaben zur Nutzung und Bewertung der fachlichen Präsenzbestände zu gewinnen, da die Medien nicht durch eine Ausleihstatistik erfasst werden. Die aktuelle Nutzung der Präsenzbestände sowie der Bedarf an unterschiedlichen Formen von Lese- und Lernplätzen und weiteren technischen Angeboten standen somit im Fokus der Befragung.

Den Benutzern standen hierfür eine Online- und Papierversion des Fragebogens zur Auswahl. Die Online-Version des Fragebogens wurde mit der Software "Enterprise Feedback Suite" (EFS) erstellt, wobei auf möglichst abwechslungsreiche Antwortmöglichkeiten geachtet wurde, um die Motivation bis zum Ende der Befragung zu erhalten.

\subsection{Ergebnisse der Befragung}

Durch die Befragung konnten 660 verwertbare Fragebögen ausgewertet werden. Diese Anzahl überstieg deutlich die Erwartungen und zeigt, wie groß das Interesse der Benutzer ist, ihre Meinung zum Ausdruck zu bringen sowie die Weiterentwicklung der Bibliothek mitzugestalten. Im Folgenden können die Ergebnisse der Befragung nur gekürzt dargestellt werden. ${ }^{7}$

\subsubsection{Teil A: Nutzerstruktur}

Beim ersten Abschnitt des Fragebogens ging es darum, die Nutzerstruktur zu erfassen, um daraus Rückschlüsse für die weiteren Angaben schließen zu können. 


\subsubsection{Nutzergruppen}

Mit 48,03\% sind Studierende die größte Nutzergruppe (Abb. 2). Es folgen Berufstätige (21,36\%) sowie Hochschulmitarbeiter und Wissenschaftler $(17,27 \%)$. Zu den Sonstigen zählen z.B. Doktoranden, Rechtsreferendare, Arbeitssuchende, Schriftsteller, Ehrenamtliche, Bibliothekare/Archivare sowie ein Kurator, Hobbyforscher und Freiberufler. Alle Angaben verdeutlichen, dass der Hauptlesesaal von der Breite der Bevölkerung genutzt wird und somit sehr unterschiedliche Interessen vertreten sind.

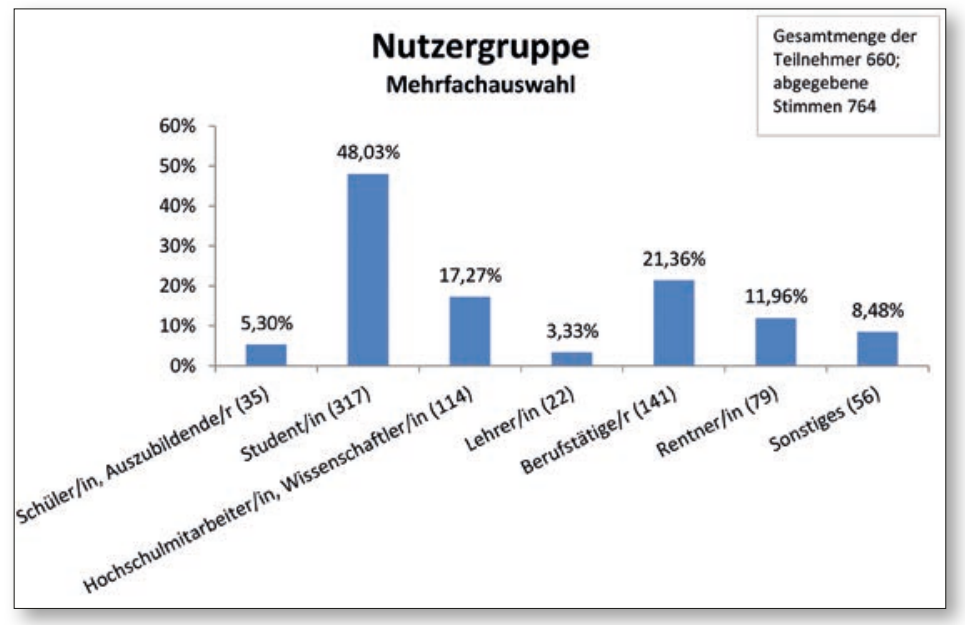

Abb. 2: Nutzergruppen des Hauptlesesaals

\subsubsection{Nutzungsgründe}

Bei der Frage nach dem Grund der Lesesaalnutzung wurden häufig mehrere Antworten angegeben (Abb. 3). Mit 51,51\% wird das Studium als häufigster Nutzungsgrund genannt, was mit der größten Nutzergruppe der Studierenden korreliert. Weitere Gründe sind Forschung (36,06\%), privates Interesse $(29,84 \%)$ und berufliches Interesse $(23,33 \%)$.

Bei Sonstiges werden u.a. genannt: ScannerNutzung, Presse, Familienforschung, Bewerbungen schreiben, Ruhe für konzentriertes Arbeiten, Zeitungslesen und ehrenamtliche Tätigkeit. Um allen Interessen entsprechen zu können, sollten die Angebote in unterschiedlicher Tiefe der Fachdisziplinen vertreten sein, d.h. einfach verständliche Einführungen für Schüler, Studienliteratur für Studierende, praxisorientiere Werke für Berufstätige sowie Quellen und zentrale Nachschlagewerke für Forschung und Lehre.

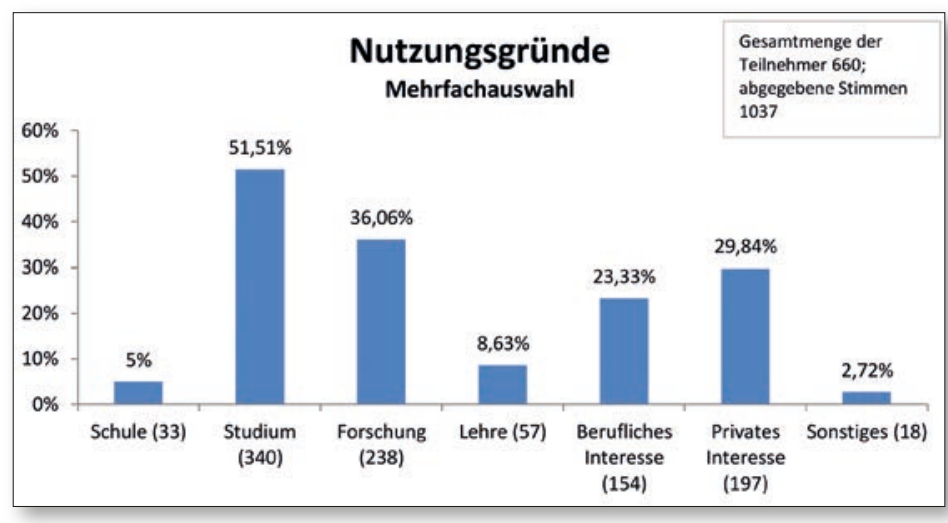

Abb. 3: Nutzungsgründe zum Arbeiten im Hauptlesesaal

\subsubsection{Teil B: Nutzungsverhalten}

Dieser Teil der Befragung richtet sich nach den Zeiten und der Häufigkeit, in denen der Hauptlesesaal genutzt wird. Im Interesse steht auch der Bedarf nach weiteren Öffnungszeiten.

\subsubsection{Nutzungszeiten}

Der Hauptlesesaal ist am stärksten von 13 bis 17 Uhr genutzt. Donnerstags bis samstags nimmt die Nutzung nochmals leicht zu. Viele Benutzer halten sich dabei länger in der Bibliothek auf, so dass neben der Infrastruktur des Lesesaals auch ansprechend gestaltete Pausen- und Erholungsbereiche gewünscht werden.

\subsubsection{Nutzungshäufigkeit}

Viele der befragten Benutzer arbeiten sehr regelmäßig im Hauptlesesaal (Abb. 4): fast jeder Zweite (49\%) mindestens einmal in der Woche. Nur $14 \%$ der Benutzer kommen weniger als einmal im Monat. Studierende kommen im Jahresverlauf mit unterschiedlicher Frequenz je nach Prüfungsphase oder zum Schreiben wichtiger Arbeiten. Da sich die Anforderungen im Verlauf eines Studiums verändern, sind verschiedene Bereiche für kommunikative Gruppenarbeit und konzentrierte stille Einzelarbeit nachgefragt.

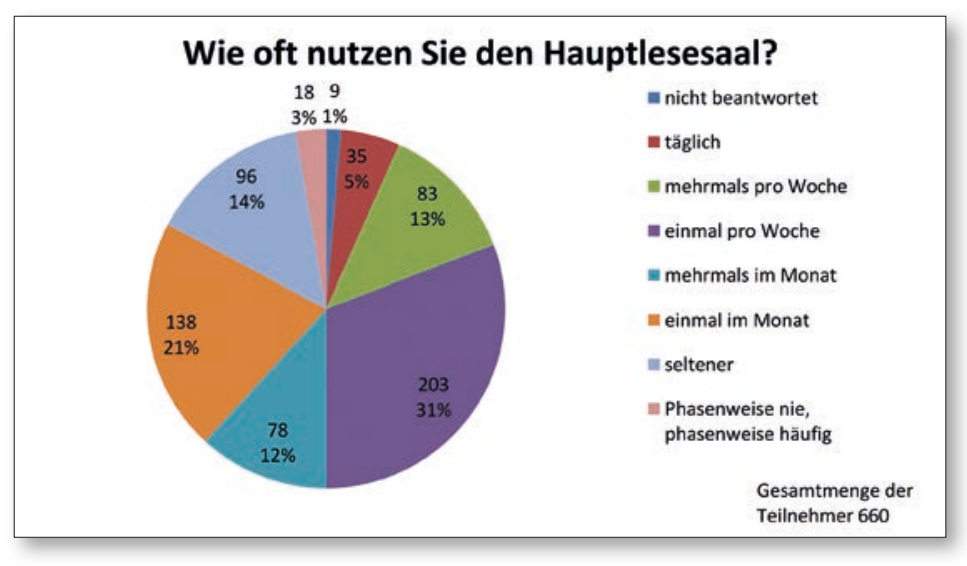

Abb. 4: Häufigkeit der Nutzung 


\subsubsection{3 Öffnungszeiten}

Die Frage nach dem Bedarf weiterer Öffnungszeiten richtet sich vor allem darauf, diese mit dem Erweiterungsbau auszudehnen. Die Mehrzahl der befragten Benutzer (56\%) bewertet die Öffnungszeiten als ausreichend (Abb. 5). Es gibt jedoch sehr viele Benutzer (43\%), die sich eine Ausweitung wünschen. Diesen zusätzlichen Bedarf sollte die Bibliothek im Blick behalten.

\section{Sind die Öffnungszeiten ausreichend?}
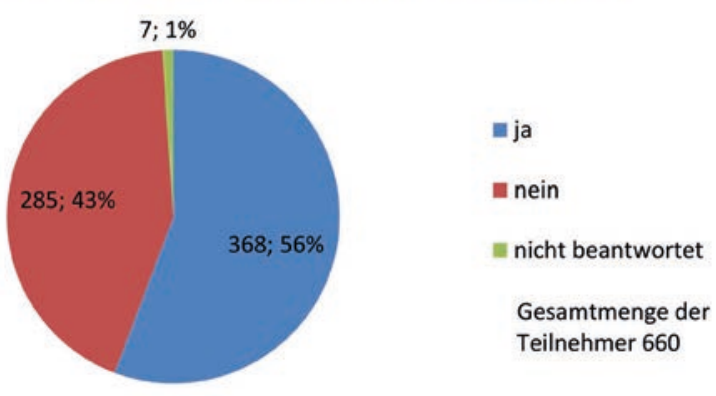

Abb. 5: Bewertung der Öffnungszeiten

Die Tabelle (Abb. 6) stellt die Angaben der Benutzer dar. Sie zeigt, dass vor allem der Samstagnachmittag und auch verlängerte Abendzeiten gewünscht werden. Auch sonntags sollte die Bibliothek für einige geöffnet sein.

\subsubsection{Teil C: Präsenzbestände}

Dieser Teil beschäftigt sich mit der Nutzung des Präsenzbestands der Fächer sowie anderer Materialien, die im Lesesaal genutzt werden.

\subsubsection{Arbeitsmaterialien}

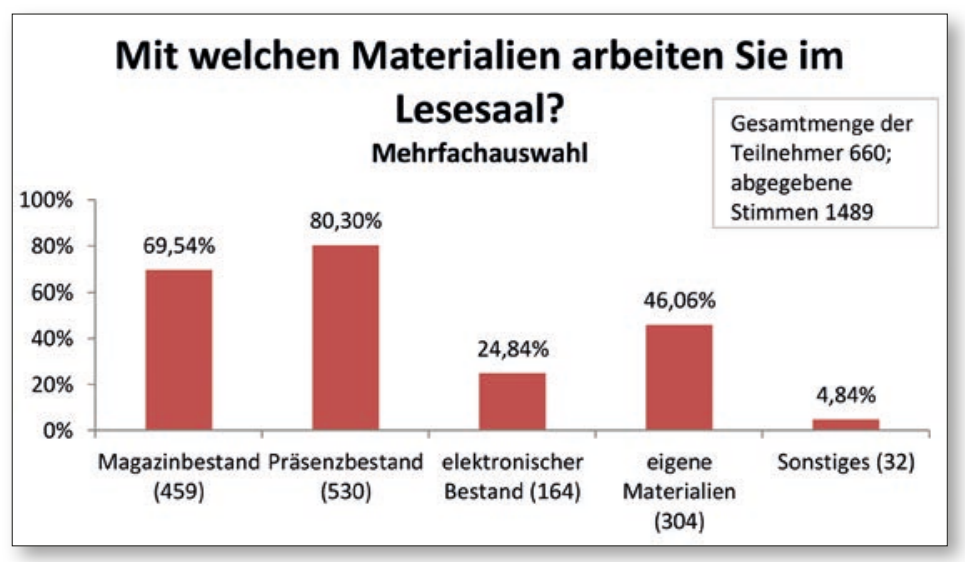

Abb. 7: Arbeitsmaterialen im Hauptlesesaal

Die Frage nach den Arbeitsmaterialien zielt darauf $a b$, die Nutzung des Präsenzbestands im Vergleich mit anderen Arbeitsmaterialien einzuordnen (Abb. 7).

Mit Abstand sind die Präsenzbestände der Hauptgrund $(80,30 \%)$, im Hauptlesesaal zu arbeiten. Auch die Bestellungen aus dem Magazin (69,54\%) spielen eine wichtige Rolle, da diese überwiegend eine Ausleihbeschränkung auf den Lesesaal haben. Etwa die Hälfte der Benutzer (46,06\%) nutzt (auch) eigene Materialien wie Bücher, die mitgebracht werden. Bei den Angaben zu Sonstiges wurden u.a. Zeitungen, Fernleihen und das WLAN der Bibliothek genannt. Anhand dieser Angaben kommt dem Präsenzbestand eine hohe Bedeutung zu und berechtigt, diesen auszubauen und zu verbessern. Auch in den offenen Kommentaren der Benutzer kommt dies verstärkt zum Ausdruck (z.B. durch konkrete Titelvorschläge).

\begin{tabular}{|l|r|r|}
\hline Werktags (bisher Mo-Fr, 8-20 Uhr) & früher & 8 \\
\hline Wochenende (bisher nur Sa, 9-13 Uhr; So geschlossen) & später & 107 \\
\hline & früher & 15 \\
& später & 226 \\
\hline Besondere Öffnungszeiten & Sonntagsöffnung & 81 \\
\hline generell erweiterte Öffnungszeiten & Sa in den Ferien & 2 \\
\hline Sonderlesesaal & Mo-So, 8-24 Uhr oder 6-22 Uhr & 2 \\
\hline
\end{tabular}




\subsubsection{Fachgebiete}

Die Zuordnung der Benutzer zu ihren Fachgebieten (Abb. 8) zeigt, dass die Schwerpunktfächer der Bibliothek sehr gut genutzt werden: Geschichte mit Zeitgeschichte (35,3\%), Allgemeine Nachschlagewerke $(33,18 \%)$, Jura $(21,81 \%)$, Wirtschaftswis$(21,21 \%)$. Für diese Fächer wurde von den Fachreferenten mehr Platz für weitere Bände gewünscht, was dem Profil der Bibliothek und der Ausleihstatistik für Magazinbände entspricht. Ein Ausbau der Präsenzbestände sollte mit zusätzlichen Räumlichkeiten umgesetzt werden. senschaften $(21,66 \%)$ und Baden-Württemberg

sich jedoch, dass in vielen Fächern ein größerer Bestand und Literatur zu neuen Forschungsgebieten gewünscht wird.

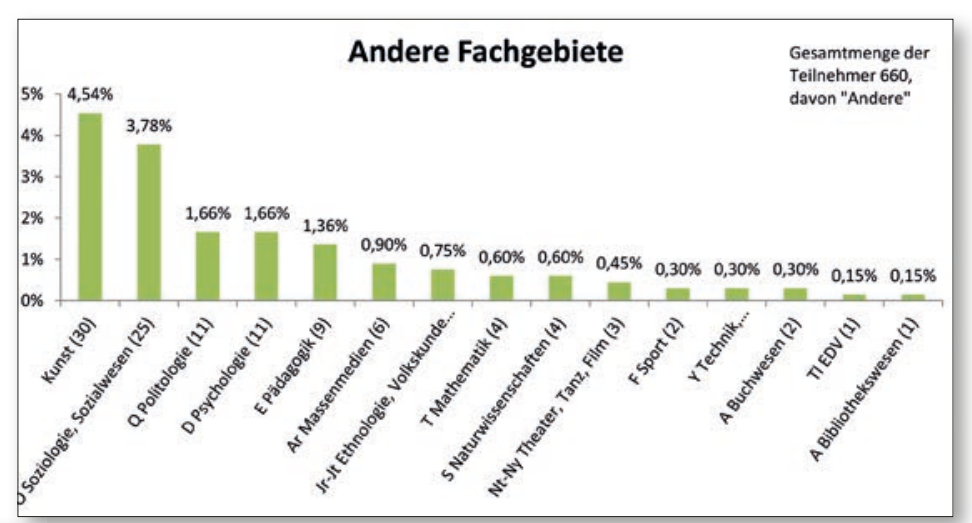

Abb. 10: Zuordnung der Benutzer zu weiteren Fachgebieten

\subsubsection{Bewertung der Präsenzbestände}

Die Gesamtbewertung der Präsenzbestände fällt sehr positiv mit überwiegend "gut" bis "sehr gut" aus. Hier wurden folgende Kriterien abgefragt:

- Literatur zu aktuellen Themen und neuen Forschungsgebieten $(50,29 \%)^{8}$,

- Aktualität der Auflagen (54,99\%),

- Breite des Angebots zu unterschiedlichen Teildisziplinen des Fachs (46,21\%),

- Klassiker des Fachs/Primärquellen zur historischen Forschung $(52,72 \%)$,

- Angebot an Hand- und Lehrbüchern (51,52\%).

Die Fächer der Sprach- und Literaturwissenschaften (K/L) wurden nochmals genauer ausgewertet (Abb. 9). Am stärksten wird die Germanistik nachgefragt.

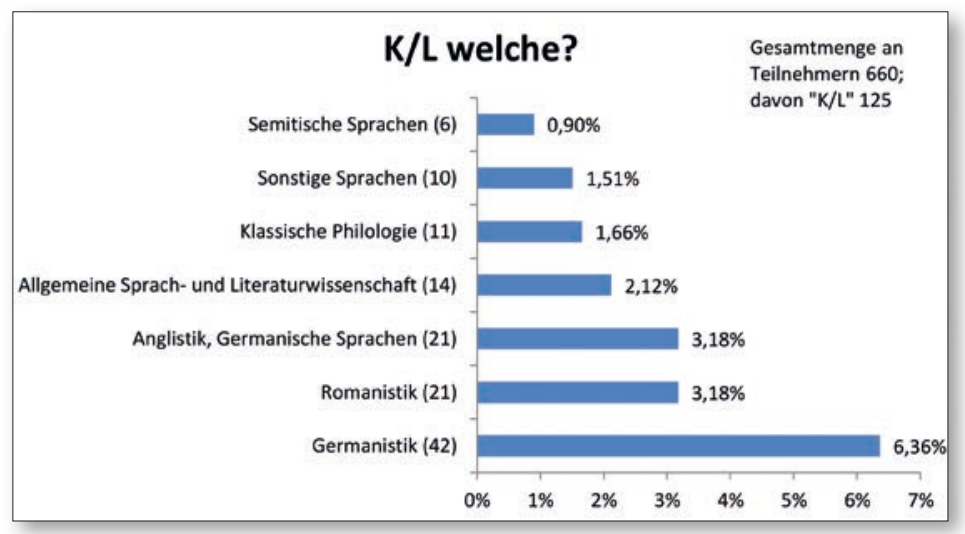

Abb. 9: Nutzung der Präsenzbestände der Sprach- und Literaturwissenschaften

Die weiteren Fächer zeigen eine geringere Nutzung. Ein wesentlicher Grund dafür ist der kleine Präsenzbestand, der damit weniger nachgefragt wird. Aus den Kommentaren der Benutzer zeigt
Dazu ist anzumerken, dass ca. 19 - 23\% der Benutzer bei jedem Kriterium keine Angaben gemacht haben, so dass sich dadurch die positiven Angaben nochmals verstärken.

Das Diagramm (Abb. 11) fasst die Angaben übersichtlich zusammen:

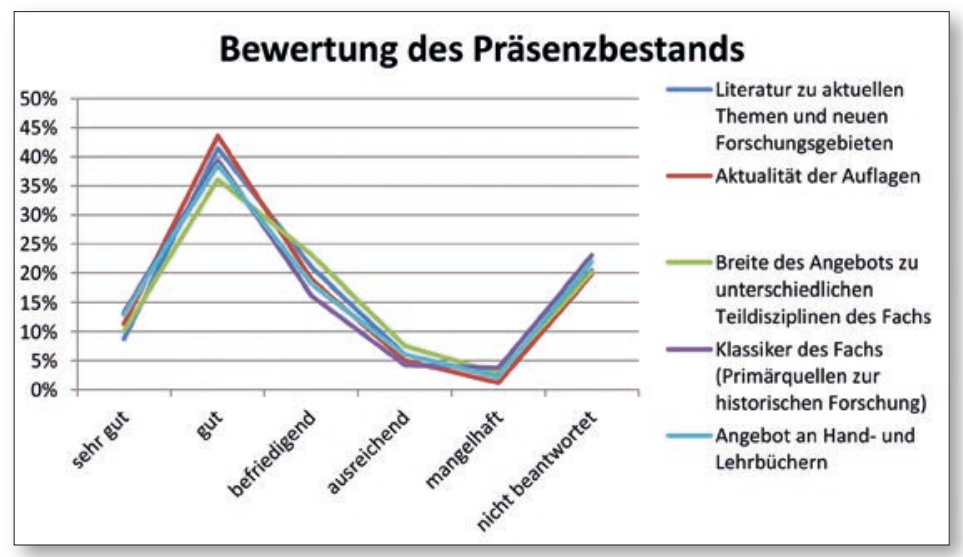

Abb. 11: Bewertung der Präsenzbestände des Hauptlesesaals mit "gut" und "sehr gut". 


\subsubsection{Lehrbuchangebot}

Das Lehrbuchangebot bewerten $41,81 \%$ als ausreichend. Insgesamt wünschen sich 40\% mehr Lehrbücher. ${ }^{9}$ Handlungsbedarf zeigt sich in den Benutzerkommentaren besonders bei Jura und den Wirtschaftswissenschaften. Trotz des bereits großen Präsenzbestandes besteht die Nachfrage nach weiteren Lehrbüchern.

\subsubsection{Online-Angebote}

Eine interessante Frage richtet sich nach der Akzeptanz von Online-Angeboten, wenn parallel eine gedruckte Ausgabe vorliegt. Die Mehrheit der befragten Benutzer (70\%) bevorzugt weiterhin noch die gedruckte Ausgabe. Dagegen sprechen sich $24 \%$ für Online-Ressourcen aus. ${ }^{10}$ Dieser Bereich sollte weiterhin im Fokus bleiben, um Nutzern einen Umstieg auf neue elektronische Ressourcen zu erleichtern. Es bestehen hierbei auch große Unterschiede bei den Fächern.

\subsubsection{Teil D: Technische Ausstattung}

Die technische Ausstattung des Hauptlesesaals ist wesentlicher Bestandteil der Infrastruktur zum Arbeiten in der Bibliothek. Zu vielen Angeboten wurde nicht von allen eine Aussage gemacht. Es zeigte sich jedoch, dass insbesondere das WLAN verbessert werden sollte (Abb. 12). Diese Anregung wurde im Juli 2018 umgesetzt. stehen, die für weitere Arbeitsplätze zur Einzelund Gruppenarbeit sowie für Pausen- und Entspannungsbereiche eingerichtet werden können. Vor diesem Hintergrund sind die Aussagen und Wünsche der Benutzer eine wichtige Planungsgrundlage.

\subsubsection{Arbeitsatmosphäre im Hauptlesesaal}

In der Zeit der Befragung führte der Baustellenlärm des Erweiterungsbaus zu Beeinträchtigungen. Dennoch wird der Lesesaal von 72,41\% der Benutzer mit "gut" und "sehr gut" als ruhiger Raum für konzentriertes, ungestörtes Arbeiten bewertet (Abb. 13).

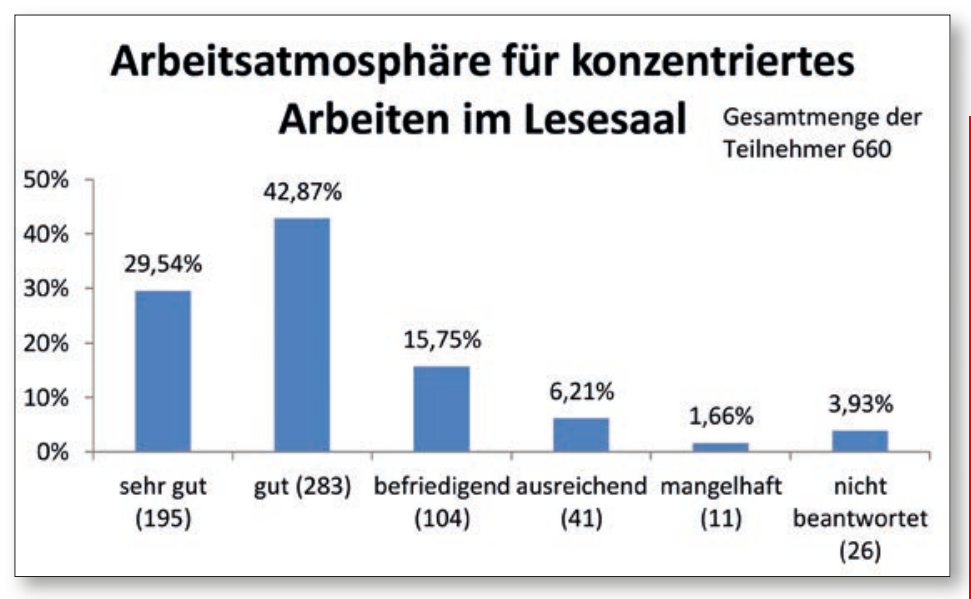

Abb. 13: Arbeitsatmosphäre im Hauptlesesaal

\begin{tabular}{|l|r|r|r|r|}
\hline & $\begin{array}{r}\text { Sehr gut } \\
\text { und gut }\end{array}$ & $\begin{array}{r}\text { Befriedigend } \\
\text { und ausreichend }\end{array}$ & Mangelhaft & $\begin{array}{r}\text { Keine Angabe/ } \\
\text { keine Nutzung }\end{array}$ \\
\hline Recherche-PCs & $49,99 \%$ & $17,11 \%$ & $1,51 \%$ & $31,36 \%$ \\
Kopierer & $42,41 \%$ & $26,06 \%$ & $4,24 \%$ & $27,27 \%$ \\
Buchscanner & $41,51 \%$ & $16,96 \%$ & $2,87 \%$ & $38,63 \%$ \\
Mikroformen-Lesegeräte & $17,56 \%$ & $8,32 \%$ & $2,57 \%$ & $71,51 \%$ \\
Mikroformen-Scanner & $14,84 \%$ & $5,9 \%$ & $1,66 \%$ & $77,57 \%$ \\
Vergrößerungsgerät & $11,96 \%$ & $4,84 \%$ & $0,75 \%$ & $82,42 \%$ \\
WLAN & $31,96 \%$ & $19,84 \%$ & $13,03 \%$ & $35,15 \%$ \\
LAN & $18,93 \%$ & $9,08 \%$ & $4,69 \%$ & $67,27 \%$ \\
Steckdosen & $45,89 \%$ & $18,17 \%$ & $6,81 \%$ & $29,09 \%$ \\
\hline
\end{tabular}

Abb. 12: Bewertung der technischen Ausstattung

\subsubsection{Teil E: Arbeitsatmosphäre und Lernumgebung}

Dieser Teil der Befragung beschäftigt sich mit der Bewertung der Arbeitsatmosphäre für konzentriertes Arbeiten, wofür der Hauptlesesaal vorgesehen ist. Darüber hinaus werden durch die baulichen Veränderungen neue Flächen zur Verfügung 


\subsubsection{Optimale Lernumgebung}

Grundsätzlich besteht ein großer Bedarf an ruhig gelegenen Einzelarbeitsplätzen (89,39\%), wie dies das derzeitige Konzept des Hauptlesesaals vorsieht. Einige Benutzer (8,78\%) wünschen sich darüber hinaus noch eine Laptop-freie Zone und/oder Arbeitskabinen zum abgegrenzten Arbeiten (25\%). Da durch die Befragung überwiegend Personen angesprochen wurden, die im Hauptlesesaal arbeiten, können sich bei einer allgemeinen Befragung aller Benutzer die Angaben zugunsten der kommunikativen Zonen noch verändern. Es zeigt sich jedoch sehr deutlich, dass ergänzend zu dem ruhigen Bereich des Hauptlesesaals kommunikative Bereiche gewünscht werden. Dazu zählen Einzelarbeitsplätze in kommunikativer Arbeitsatmosphäre $(10,15 \%)$, Gruppenarbeitsräume $(38,18 \%)$ und offene Gruppenbereiche $(35,45 \%)$, wie sie derzeit teilweise schon im Informationsbereich angeboten werden. Diese Angebote sollten u.a. mit Computerarbeitsplätzen (35,3\%), einer Leseecke mit Zeitungen (36,81\%), barrierefreien Arbeitsplätzen $(20,6 \%)$, einem Entspannungsbereich $(38,48 \%)$ und Sitzmöglichkeiten im Außenbereich (48,33\%) erweitert werden.

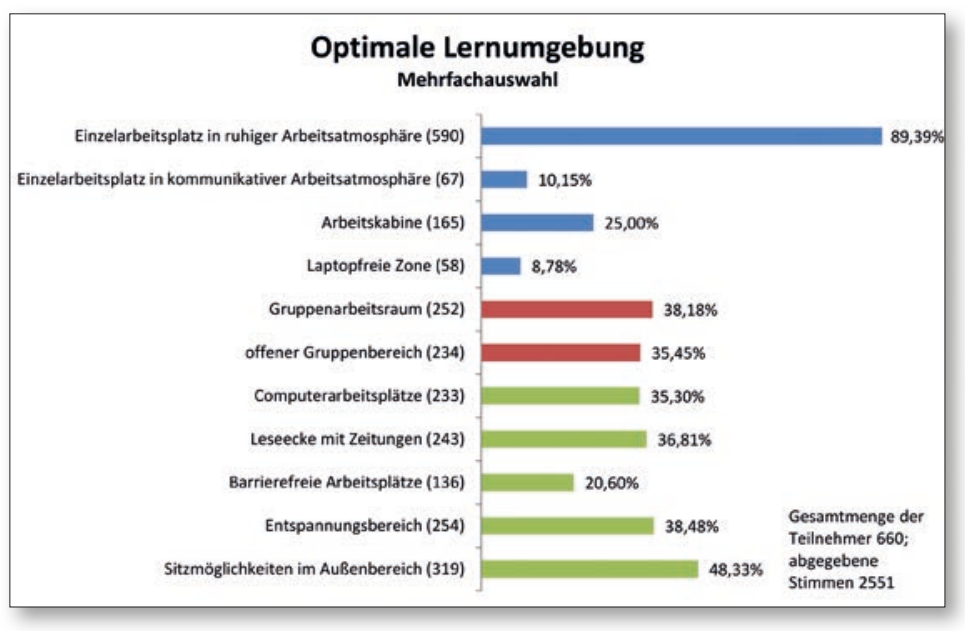

Abb. 14: Gewünschte Lernumgebung für die Zukunft

\subsubsection{Teil F: Allgemeines Feedback}

Zum Abschluss hatten die Benutzer noch die Möglichkeit Lob, Kritik, Wünsche und Verbesserungsvorschläge in einem letzten Freitextfeld einzutragen. Die vielen Einträge zeigen, dass die Benutzer sich intensiv mit den Angeboten der Bibliothek auseinandersetzen und Interesse haben, sich bei den Planungen für die Weiterentwicklung der Bibliothek einbringen zu können. Aufgrund der Menge der sehr unterschiedlichen Meinungen kann an dieser Stelle leider nicht näher darauf eingegangen werden. Zwei Beispiele sind exemplarisch im Kasten auf Seite 19 abgebildet. Sie werden aber in die weiteren Entscheidungen der Bibliotheksleitung einbezogen.

\section{Fazit und Anregungen}

Ziel der Ist-Analyse des Hauptlesesaals (mit der Befragung der Fachreferenten und den Bestpractice-Vergleichen anderer wissenschaftlicher Bibliotheken) sowie der detaillierten Benutzerbefragung ist es, die derzeitige Nutzungssituation des Hauptlesesaals aus unterschiedlichen Gesichtspunkten zu analysieren und mit den Bewertungen und Wünschen der Benutzer abzugleichen, um für die Zukunft eine Basis für weitere Planungen zu gewinnen. Aus den vielen Angaben der Benutzer zeigt sich, dass mit dem bisherigen Konzept des Hauptlesesaals weitergearbeitet werden kann und die Angebote der Bibliothek überwiegend in allen Bereichen gut bewertet werden. In einzelnen Bereichen wird aber deutlich, dass die Kommentare der Benutzer wertvolle und wichtige Anregungen bieten, die zu Verbesserungen und neuen Angeboten führen sollten.

Viele Benutzer wünschen sich eine Erweiterung der Öffnungszeiten besonders samstags und in den Abendzeiten. Im Konzept des Erweiterungsbaus ist bereits vorgesehen, mit geringerem Personaleinsatz die Bibliothek länger öffnen zu können. Die detaillierten Benutzerangaben können dabei eine hilfreiche Planungsgrundlage sein.

Die Nutzung der Präsenzbestände wird zu 80\% bestätigt, so dass ein Ausbau der Fächer angestrebt werden sollte. Dies ist dringend erforderlich, da über viele Jahre keine Veränderungen mit den begrenzten räumlichen Verhältnissen möglich waren. Grundlage für weitere Überlegungen 
bieten dazu die Kommentare der Benutzer. Bedarf wird nahezu bei allen Fächern mit konkreten Vorschlägen angemeldet. Da 40\% der Benutzer mehr Lehrbücher wünschen, sollte verstärkt auch dieser Bestand ausgebaut werden. Soweit es die Finanzierung über die Fachetats zulässt, sollte ergänzend ein weiteres Exemplar für die Ausleihe beschafft werden.

Die große Mehrheit der Benutzer $(70 \%)$ zieht weiterhin gedruckte Ausgaben den elektronischen Ressourcen vor. Da Online-Ausgaben vielfältige Recherchemöglichkeiten erlauben, sollte jedoch der elektronische Bestandsaufbau nicht vernachlässigt werden. Hier ist zu überlegen, wie Benutzern der Zugang zu OnlineRessourcen erleichtert werden kann. Eine wesentliche Voraussetzung ist hierfür eine gute Internetverbindung. Insbesondere beim WLAN wurden von den Benutzern Verbesserungen gewünscht, aber auch mehr Rechercheplätze in der Nähe der Präsenzbestände könnten die Situation verbessern. Die WLB hat hierfür bereits erste Maßnahmen ergriffen und das WLAN auf ein neues Angebot umgestellt.
Erfreulich ist, dass der Erweiterungsbau sowie eine Neukonzeption der Bibliothek sehr positiv aufgenommen werden. Viele Benutzer wünschen sich für die Zukunft vielfältigere Lernumgebungen, die je nach Studienphase und individueller Arbeitssituation ausgewählt werden können. Ein differenziertes Angebot an Einzelarbeitsplätzen und Bereichen zum kommunikativen Lernen sollte dabei geschaffen werden. Gewünscht werden hierbei helle Räume und freundliche Lernumgebungen auch in der Farbgestaltung. Da viele sich längere Zeit in der Bibliothek aufhalten, werden Pausenbereiche (z.B. Cafeteria, Zeitungsleseecke) sowie Entspannungsbereiche mit entsprechendem Mobiliar immer wichtiger. Auch für diese Anliegen verspricht der Erweiterungsbau entsprechende Angebote. Wesentlich für die kommenden Jahre wird jedoch sein, das Bestandsgebäude möglichst im Anschluss an den Umzug in den Erweiterungsbau zu sanieren, um weitere Flächen für noch offen bleibende Wünsche bieten zu können.

\section{Christine Herrmann}

